\title{
Preoperative Education System to Assist Patients Undergoing TAVI Surgery: A Digital Health Solution
}

Ali Naserian Mojadam ${ }^{1 *}$, Najaf Nadeem ${ }^{2}$, Hussein Beydoun ${ }^{2}$, Samina R Abidi ${ }^{3}$, Ali Rizvi ${ }^{3}$ and Syed SR Abidi ${ }^{1}$

${ }^{1}$ Department of Health Informatics, Faculty of Computer Science, Dalhousie University, Canada

${ }^{2}$ Department of Cardiology, QEII Health Sciences Centre, Canada

${ }^{3}$ Department of Medical Informatics, Dalhousie University, Canada

\begin{abstract}
Personalized patient education is a critical factor in the healthcare service delivery as it contributes to better management of diseases and ultimately enhanced clinical outcomes. Trans-Catheter Aortic Valve Implantation (TAVI) is a standard cardiac procedure for patients with severe aortic stenosis. TAVI is a minimally invasive procedure, however; this procedure is still associated with considerable risks based on the condition of the patient before performing TAVI. To obtain an informed consent, it is important to appropriately inform patients and their families about the risks and considerations associated with TAVI. Our research objective is to leverage digital health technologies to develop a personalized preoperative patient education system for TAVI patients. An iterative user-Centered approach was used to develop "TAVI System", and its knowledge content is based on digitized evidence and clinical expertise. We used a knowledge management approach to model, computerize, and integrate TAVI patient assessment to generate personalized educational messages.
\end{abstract}

Keywords: Cardiology; Digital health; Health informatics; Personalized patient education; TAVI

\section{Introduction}

Trans-Catheter Aortic Valve Implantation (TAVI) is an innovative surgical procedure that is offered to patients who are considered high risk for open heart surgery (especially to treat severe aortic stenosis) due to advanced age and multiple comorbid conditions [1-3]. Although, TAVI is a minimally invasive procedure, it is still associated with considerable risks depending on the condition of the patient $[2,3]$ Therefore, when seeking an informed consent for the surgery, it is both important and essential to inform patients and their families about the benefits and risks associated with TAVI, so that patients and their families can make an informed decision about whether to undergo the TAVI procedure [3]. Preoperative interactions with patients are intended to provide detailed information about TAVI related treatment strategies and the associated risks [4]. However, it is noted that these information sessions are not effective for a number of reasons, such as the most healthcare providers have limited time during the clinical encounter to comprehensively explain the procedure and associated risks [5-7], patients are stressed at that point and hence do not fully comprehend and remember the information after leaving the hospital [8], and since patient's risk tolerance and expectations vary, the generic information presented to them is not deemed pertinent and sufficient by patients $[9,10]$. The prevailing limitations with regards to patient education and a communication gap between patients and providers is noted to affect patient satisfaction [11-14].

Patient satisfaction is related to understanding of their condition and the proposed medical interventions [11-13]. Patients are generally anxious prior to major surgery and if they are properly informed about the surgical procedure, it reduces their preoperative anxiety which in turn improves the outcomes of the surgical procedure $[15,16]$. A patient-cantered care approach [14] to reduce patient anxiety, involves engaging the patient in shared decision making regarding therapeutic choices, and educating them about their condition, the procedure and their follow-up plan through personalized educational content $[13,15]$.
In our research, we focus on developing a digital health based shared decision-making environment to assist patients who are recommended the TAVI surgical procedure. Taking a patient-centred approach, our objective is to provide comprehensive and personalized patient education material to help patients (and their families) learning about the TAVI procedure and its suitability with respect to their needs and expectations. In this paper we present a digital health based preoperative personalized patient education system that offers the following functionalities: (a) Assist in TAVI patient assessment with respect to a 2 local patient assessment protocol to identify associated risks and specific procedural considerations for each patient with respect to the TAVI procedure; (b) Establish a shared decision making environment in which the based on the observed risks, the specialist and patient determine the intervention options; (c) Select and deliver patient-specific educational content, based on the patient's TAVI assessment and intervention choice, to help patients prepare for the TAVI procedure. We evaluated our TAVI patient education system based on a large volume of clinical scenarios, to determine its ability to assess the risks and select patient-specific educational content.

\section{Research approach}

Given our aim to engage and educate TAVI patients, our patient education approach is based on the Patient-Centered Care (PCC) Model [17] that supports informed patients to improve shared decision making. The educational content is personalized in line with the

*Corresponding author: Ali Naserian Mojadam, Department of Informatics, Dalhousie University Halifax, Nova Scotia, 3471 Dutch Village Rd, Apt 1102, Canada, Tel: (902)-830-8301; E-mail: AL553930@dal.ca

Received May 21, 2018; Accepted May 28, 2018; Published June 02, 2018

Citation: Mojadam AN, Nadeem N, Beydoun H, Abidi SR, Rizvi A, et al. (2018) Personalized Preoperative Education System to Assist Patients Undergoing TAVI Surgery: A Digital Health Solution. J Health Med Informat 9: 313. doi: 10.4172/21577420.1000313

Copyright: ( 2018 Mojadam AN, et al. This is an open-access article distributed under the terms of the Creative Commons Attribution License, which permits unrestricted use, distribution, and reproduction in any medium, provided the original author and source are credited. 
patient's associated risks and procedural considerations to increase their knowledge about the procedure and enhance their confidence to express their health concerns and preferences in shared decision making [18]. To fully understand the current clinical workflow for pre-operative TAVI patient education, our research approach involved shadowing the TAVI team at the Queen Elizabeth II (QEII) hospital. The shadowing experience was conducted for three weeks, for a span of two days each week. During this shadowing period, we shadowed five new TAVI patients during their first clinical visits and three followup TAVI patients. The shadowing step enabled to learn first-hand how the TAVI team provided TAVI related information to new and followup patients, and what were the limitations of the current process. The current patient education process is led by a TAVI nurse and it included providing verbal patient education, showing a short video about TAVI procedure, and answering patient's questions. Most of the information about the TAVI procedure was generic in nature, no written patient education materials was provided for the patient to take home.

In order to suggest potential improvements to the current TAVI patient education process, our approach was to (i) illustrate the complete TAVI process, (ii) identify PCC specific patient education opportunities within the TAVI process in terms of a process model, and (iii) implement patient education components at the strategic points in the process to achieve maximum shared decision making and learning by patients. With data gathered from the shadowing exercise, we developed a business process model of the current TAVI patient assessment and decision-making process using the Business Process Modelling Notation (BPMN). The BPMN based TAVI process model (Figure 1) illustrates the constituent patient assessment tasks, the roles of the different actors, the decision points in the process and the information objects used at different points in the TAVI process.

We applied a health knowledge management approach [19] to computerize the educational content and the personalization strategy. In essence, the design of TAVI patient education system includes: (a) a knowledge model that comprises a set of decision rules representing the relationship between patient assessments, needs, preferences (as input) and corresponding educational content (as output); and (b) a knowledge processing engine that takes as input the patient's parameters and generates as output a personalized patient education document which comprises a collection of individual educational documents that are selected and amalgamated to yield a coherent and personalized educational resource for the patient. The knowledge modeling approach guided the acquisition, representation, inferencing and transfer of the TAVI patient education knowledge as follows:

- Knowledge acquisition: This process involves identifying the domain knowledge required to accurately and competently asses a patient and to generate a personalized educational document $[19,20]$. In our system, the main source for knowledge was interventional cardiologists and nurses with whom the team had multiple meetings

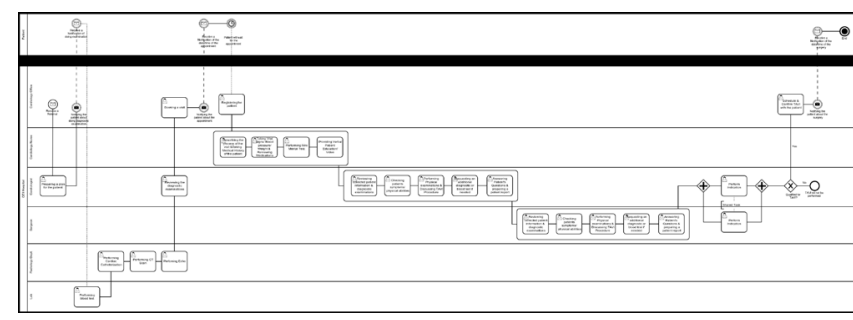

Figure 1: BPMN diagram: The current preoperative clinical pathway for TAVI. to glean knowledge about the TAVI process. In addition, we also considered the limited educational pamphlets for patients undergoing surgery. After gathering the knowledge required for our system, we have to organize the knowledge in a systematic way to be able to proceed to the next process of knowledge management.

- Knowledge representation: This process involves modeling the domain knowledge in terms of a set of decision rules that can be computerized to draw inferences based on the input data [20]. In our system, "IF-THEN" rules were used for knowledge representation. We used these rules to link specific risks and considerations with the patient educational content $[20,21]$.

- Knowledge inferencing: In this process, a logic-based reasoning engine reasons over the computerized knowledge to draw inferences based on the input. In our system, when the user of the TAVI system provides the patient information, the reasoning engine examines the set of decision rules to infer an output which in this case is a collection of information items that relate to the patient parameters [22].

- Knowledge transfer: This process involves reporting the final decision/recommendation, which should concur with an expert's decision, to the user of the system [20]. In our system, the user is provided with a list of potential risks/considerations for a TAVI patient and recommends a number of personalized educational messages based on the results of patient assessment.

\section{TAVI System development}

The TAVI patient education system is designed to be used during patient's clinical visit to the TAVI clinic. TAVI team members examine the patient and enter patient information into the system. The collected patient information (as per the TAVI assessment protocol comprising a wide range of patient clinical and risk variables) is used for patient assessment and our system determines the patient's risks and highlights the therapeutic considerations-the findings are documented in a patient report that is used by the TAVI team to determine the eligibility of the patient for the TAVI procedure. Based on the system generated patient report, our system generates a personalized patient education document. The patient education document will contain generic and personalized messages based on the results of the assessment. The personalized patient education document focuses on informing patients about their conditions, risks associated with the procedure, and possible outcomes.

TAVI System is a rule-based system. The user, a TAVI team member, is presented with a patient assessment, in the interactive window, where the user has to enter the patient data into the components of patient assessment. Based on the entered data, the system searches the knowledge base for possible pattern matches according to the decision rules. "IF-THEN" rules are the main type of knowledge representation used in TAVI system. If there is a rule in the knowledge base which matches the risk variables in the patient assessment, the system identifies the possible risks/considerations and the educational content based on the patient data. The knowledge base contains educational content that should be personalized based on the rules of the system $[23,24]$. The primary source for knowledge acquisition for the TAVI System was consultations and meetings with interventional cardiologists at the QEII hospital. Figure 2 summarizes the elements of the TAVI patient education system.

Figure 3 illustrates the conceptual model of the personalized patient education system. The model contains four major elements; TAVI patient, risk variables which are included in the patient assessment, associated risks/considerations and patient education messages. In 
Citation: Mojadam AN, Nadeem N, Beydoun H, Abidi SR, Rizvi A, et al. (2018) Personalized Preoperative Education System to Assist Patients Undergoing TAVI Surgery: A Digital Health Solution. J Health Med Informat 9: 313. doi: 10.4172/2157-7420.1000313

Page 3 of 6

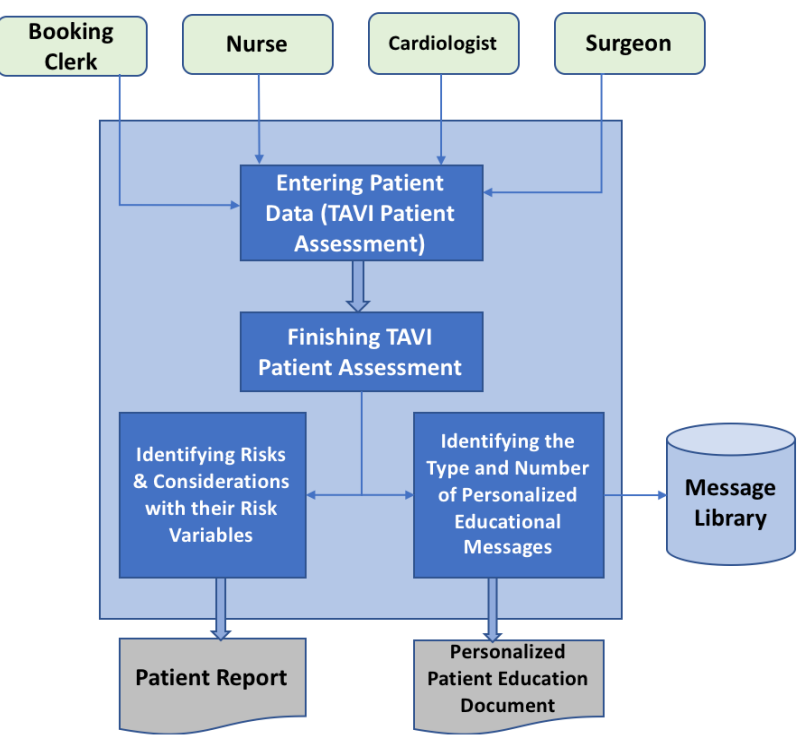

Figure 2: Workflow of TAVI system.

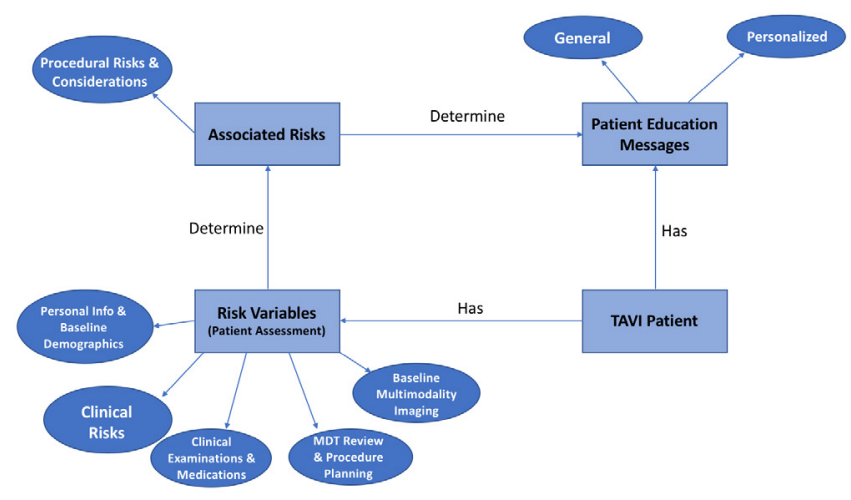

Figure 3: Conceptual model of TAVI system.

this model, each TAVI patient has risk variables in TAVI patient assessment. All the risk variables are included in the main five components of TAVI Assessment which are: Personal Information \& Baseline Demographics, Clinical Risks, Clinical Examinations \& Medications, Multidisciplinary Team Review \& Procedure Planning, and Baseline Multimodality Imaging. Furthermore, associated risks element includes preoperative risks and considerations associated with TAVI, while the patient education messages element contains the educational content of the messages which are subdivided into general and personalized messages. Risk variables element is the key for determining the number and types of preoperative risks and considerations associated with TAVI for the patient based on a set of decision rules. After determining the preoperative risks/considerations of the patient, the system will be able to identify the number and type of patient education messages that should be delivered to the patient based on another set of decision rules.

\section{TAVI system development steps}

- Step 1: Understanding and modelling the current preoperative clinical pathway: To design our system, shadowing of the TAVI team members was undertaken in September 2016 to understand the current preoperative clinical pathway for TAVI, focusing on patient education process. We developed a BPMN based current clinical pathway for TAVI. Next, based on the current workflow we identified points of patient education and presented an updated BPMN diagram (Figure 4).

- Step 2: Obtaining data collection protocol: TAVI patient assessment protocol was obtained from the TAVI team at the QEII hospital. The assessment is intended for new TAVI patients in order to assess their eligibility for a TAVI procedure. Our system has digitized the TAVI patient assessment protocol and users can use the web-based interfaces to collect the patient data and it is stored in a secure database.

- Step 3: Identifying associated preoperative considerations/ risks with their risk variables: To determine a patient's risk pertaining to the TAVI procedure, we identified a set of risk variables to determine the number and types of risks and considerations associated with TAVI procedure based on patient assessment. We identified 13 possible associated risks and considerations on the TAVI patient assessment as follows: Increase Risk of Vascular Complication, Increase Risk of Stroke, Increase Procedural Risk, Increase Risk of Pacemaker, Increase Predictor of Mortality, Needs Specific Anesthetic Consideration, Increase Prolonged Hospital Stay, Increase of Kidney Damage, Needs to Check Blood Products Availability, Active Cancer - Information Prognosis, Wait time Assessment Special Consideration, Increase Risk of Infection Endocarditis, and Eligibility for Research Study.

- Step 4: Obtaining educational content: The content of the personalized educational messages was obtained from interventional cardiologists. The focus of the educational content was driven by 10 out of the 13 risks and considerations earlier identified, thus leading to 10 categories of educational messages. Taking into account the value of the patient's risk variables we developed 19 personalized educational messages. In addition, 7 general educational messages were created for all TAVI patients. Table 1 illustrates some examples of the personalized educational messages.

Step 5: Developing decision rules: Decision rules are essential for our personalized patient education system since they are used to select the relevant patient educational content based on the patient's parameters. Two sets of decision rules were developed. The first set of rules, comprising 63 rules, determines the number and types of risks and considerations that could arise from the TAVI procedure based on the results of patient assessment. For example, if a TAVI patient has "severe pulmonary hypertension", which is one of the risk variables, then there is an identification of the risk of "increasing predictor of mortality". The system will arrive at this conclusion by analysing the 63 rules under the first set of rules.

The second set of rules, comprising 19 rules, is used to determine the number and types of personalized patient educational messages that should be delivered to the TAVI patient. The main purpose of the second set of rules is to link specific risk variables from the same patient assessment to the educational messages. For example, using the first

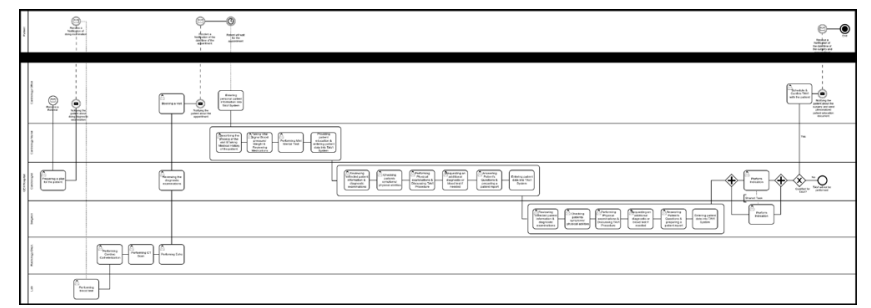

Figure 4: BPMN diagram: The proposed clinical pathway for TAVI with using TAVI system. 
Citation: Mojadam AN, Nadeem N, Beydoun H, Abidi SR, Rizvi A, et al. (2018) Personalized Preoperative Education System to Assist Patients Undergoing TAVI Surgery: A Digital Health Solution. J Health Med Informat 9: 313. doi: 10.4172/2157-7420.1000313

Page 4 of 6

\begin{tabular}{|l|l|}
\hline Risk Variable & Increase Predictor of Mortality \\
\hline General & Message Content \\
\hline Dialysis Dependent & The risk of death associated with TAVI procedure is about 1 to $2 \%$. \\
\hline $\begin{array}{l}\text { Pulmonary Hypertension OR } \\
\text { PA Systolic } \\
\text { RV }\end{array}$ & You have a significant kidney damage which is associated with increased risk of death. \\
\hline LV Angiogram OR Echo MR & $\begin{array}{l}\text { It is well known that patients with elevated systolic pulmonary artery hypertension are at increased risk from TAVI } \\
\text { procedure as the high pulmonary artery pressure is a reflection of advanced aortic valve disease. }\end{array}$ \\
\hline & The presence of significant mitral valve leakage has been associated with increased risk of adverse outcome. \\
\hline PAF & Increase risk of stroke \\
\hline $\begin{array}{l}\text { Cerebrovascular Disease OR Cerebrovascular } \\
\text { Event }\end{array}$ & $\begin{array}{l}\text { Irregular Heart Rhythm (Atrial Fibrillation): Because you have irregular heartbeat, you may be at increase the risk of } \\
\text { stroke. Overall, the risk of stroke during the TAVI procedure is about } 1 \text { to } 3 \% . \text { We will discuss with you the optimal } \\
\text { medical treatment to reduce the risk of stroke. }\end{array}$ \\
\hline $\begin{array}{l}\text { Previous Stroke: Because you have a previous stroke, you are at increased risk for subsequent stroke. Overall, the } \\
\text { reduce the risk of stroke. }\end{array}$ \\
\hline
\end{tabular}

Table 1: Personalized educational messages with their risk variables.

set of rules a patient having severe pulmonary hypertension would be identified as being at risk of mortality. Based on this determination, the second set of rules will select and deliver to the patient messages pertaining to an increasing risk of mortality due to severe pulmonary hypertension. Below are some examples of the two sets of decision rules that we used in our system:

\section{Examples from Set \#1 (63 rules):} Risk

R1: IF LVEF $=21-30 \%$ OR LVEF $\leq 20 \%$ THEN Increase Procedural

R4: IF Dialysis dependent $=$ Yes THEN Increase Predictor of Mortality 7

R6: IF Cerebrovascular disease $=$ Yes THEN Increase Risk of Stroke

R7: IF Cerebrovascular event $=$ Yes THEN Increase Risk of Stroke

Examples from Set \#2 (19 rules):

IF $\{$ R1 $\}$ THEN Message 1 (M1)

IF \{R4\} THEN Message 9 (M9)

IF $\{$ R6 + R7 $\}$ THEN Message 13 (M13)

- Step 6: Developing patient report and personalized patient education document templates: We have developed two templates to present the patient report and personalized patient education document. The patient report contains the patient's information in terms of basic identification of information, and a list of preoperative risks and considerations with their risk variables based on the results of the patient assessment. The personalized patient education document contains the main personal information of the patient as well as a list of preoperative risks and considerations with specific educational messages based on the results of the patient assessment.

Step 7: Developing the initial prototype of TAVI patient education system: In this step, the implementation of a prototype of the web-based TAVI system was undertaken. The system is developed using Java technologies. This prototype was iteratively modified by developers to match the expectations of the users and the goals of the project (Figures 5 and 6 ).

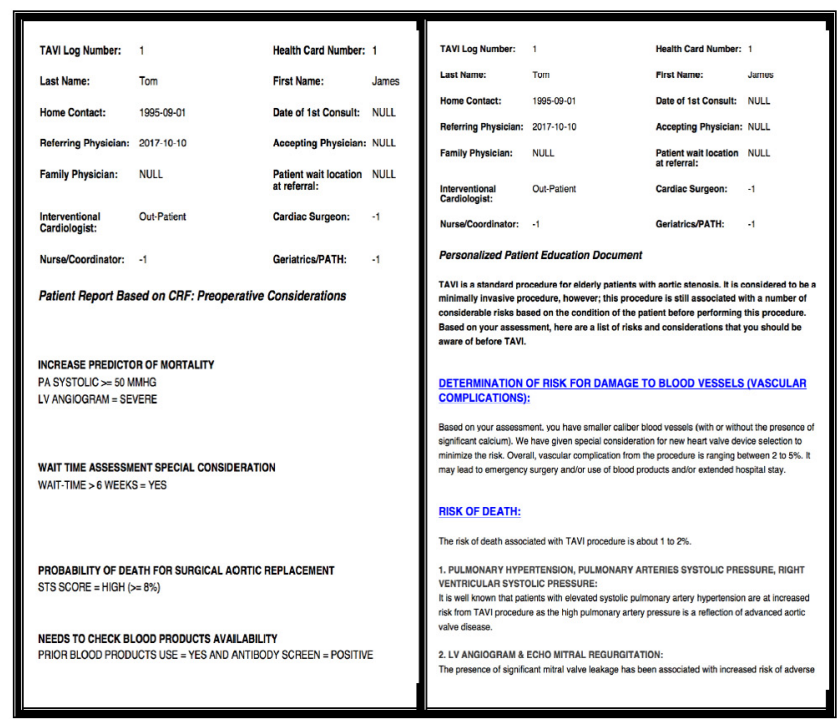

Figure 5: An example of TAVI system outputs (Patient Report and Personalized Patient Education Document).

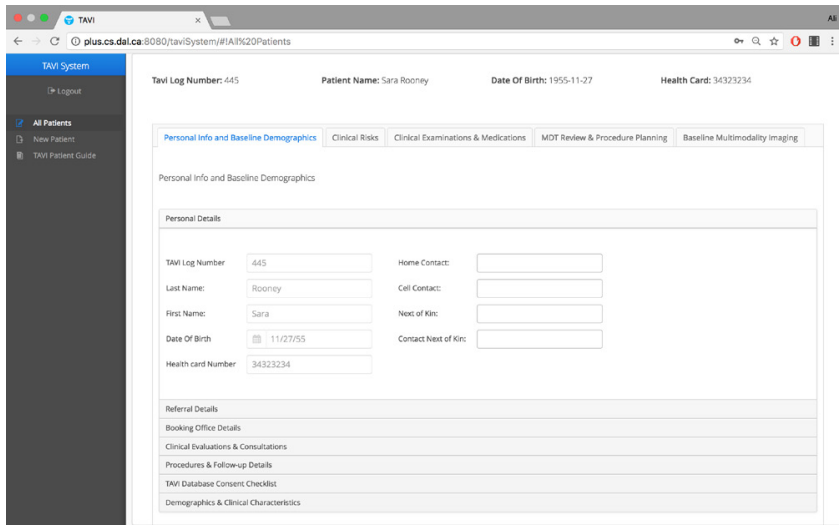

Figure 6: TAVI patient assessment interface. 


\section{System evaluation}

For evaluating the prototype, we used an internal validation method to assess the information personalization aspect of the system in terms of its ability to select the right educational messages for a given patient profile. The gold standard was the educational package recommended by the interventional cardiologists.

We used scenario-based evaluation to determine the information personalization functionality of TAVI system. For this evaluation, we prepared 40 case scenarios. We divided these case scenarios into four groups (10 case scenarios for each group) based on the largest four categories of the risks and considerations associated with TAVI procedure. The largest four categories are procedural risks, vascular complications, risk of mortality, special procedural considerations. These case scenarios were validated for clinical correctness through reviewing some literatures related to TAVI patients [25-27].

After preparing the case scenarios, we had to set up the result expectations for each case scenario. Expectations refer to the desired educational messages that should be generated by the system based on the patient profile.

Based on our evaluation, TAVI system was able to detect about $88 \%$ of the desired risk variables correctly. TAVI system failed to detect about $12 \%$ of the desired risk variables. In addition, TAVI system was able to detect approximately $80 \%$ of the desired personalized messages correctly. The system failed to detect about $20 \%$ of the desired personalized messages. This evaluation was useful to identify 11 issues with our rule-based system. All of these issues were programming issues. After identifying these issues, the development team was able to fix them. We had no issues with the knowledge content as the rules were consistent with the expert's knowledge.

\section{Discussion \& Conclusion}

Cardiac surgeries have associated risks that need to be communicated to patients so that they can make an informed consent about whether they will like to proceed with the planned procedure knowing 9 the risks. TAVI is a new surgical procedure for which general information is not as prevalent as other cardiac surgeries. Therefore, it is important to provide both relevant and comprehensive information to patients (and their families) to assist them in their decision making regarding the suitability of the procedure in concert with specialist's advice. Our patient education approach is guided by the PCC model, and we were able to implement a digital health enabled shared decision-making environment in which the care provider and patient jointly consider the risks and considerations and based on the patient's needs and preferences our system generates personalized educational content. To address the educational needs of patients, and in the process standardizing the patient data collection and assessment process, we developed a "proof-of-concept" preoperative personalized patient education system for TAVI patients who offer the following functionalities: (a) Computerization of the TAVI assessment protocol and underlying risk assessment logic to determine the associated risks for a patient. Our system presents the patient's risks and considerations in terms of an auto-generated report that is used by the TAVI team for clinical decision-making; (b) Formulation of a large set of patient educational messages that cover most topics related to TAVI surgery; and (c) Selection and delivery of personalized educational messages, based on the patient's TAVI assessment, to prepare a personalized patient education document that can educate the patient about the TAVI procedure. The personalized educational document is printed and provided to patients. We evaluated the information personalization capabilities of our system and after a round of updates; the system is now able to produce fully correct and complete patient educational documents. We conclude that this proof-of-concept system, demonstrates a digital health based solution for (a) reducing the communication gap between patients and provides; (b) reducing the anxiety of patients undergoing cardiac surgery by providing them personalized educational content that can help them understand both the procedure, its risks and expected outcomes; (c) implementing a shared decision making environment-which does not exist in surgical settings-to prepare the patient for TAVI surgery; (d) standardized patient assessment and risk evaluation based on a validated protocol; (e) enhancing the knowledge of patients so that they are in a better position to give consent to the surgical procedure; and (f) orchestrating an effective and efficient clinical workflow for the TAVI surgeries, taking into account the clinical tasks, educational opportunities, roles of TAVI team members, data sharing and coordination between the TAVI team.

The overall solution is generic and can be applied to different surgical procedures, provided surgery specific assessment protocols, educational content and information personalization logic is available. We posit that such solutions need to be instituted for complex and risk adverse surgeries so that patients are able to understand the procedure and learn about the risks and how to mitigate these risks. The provision of personalized patient education messages may reduce anxiety levels of patients which in turn will help to improve the surgical outcomes. In this research, we were unable to perform two evaluation studies which are important for establishing the clinical utility and efficacy of the proposed solution-i.e. (i) a qualitative study to gather the enduser feedback regarding the usability of the system and the utility of the personalized education content, and (ii) a mixed-methods study to evaluate the perception and outcome of the shared decision-making aspect of the solution. We plan to conduct these studies in the future.

\section{References}

1. Thomas M (2008) Trans-catheter aortic valve implantation in the United Kingdom: NICE guidance. Heart 95: 674-675.

2. Aldalati $\mathrm{O}$, MacCarthy $\mathrm{P}$, Dworakowski $\mathrm{R}$ (2017) Trans-catheter aortic valve implantation: Contemporary practice and the future. Cardiol J 24: 206-215.

3. Phan K, Wong S, Phan S, Ha H, Qian P, et al. (2013) Transcatheter aortic valve implantation (TAVI) in patients with aortic stenosis. Heart Lung Circ 24 649-659.

4. Dathatri S, Gruberg L, Anand J, Romeiser J, Sharma S, et al. (2014) Informed consent for cardiac procedures: Deficiencies in patient comprehension with current methods. Ann Thorac Surg 97: 1505-1512.

5. Coulter A (2011) Engaging patients in healthcare. Ang coult pic 1: 1-58

6. Wantland D, Portillo C, Holzemer W, Slaughter R, McGhee E (2004) The effectiveness of web-based vs. non-web-based interventions: A meta-analysis of behavioral change outcomes. J Med Internet Res 6: 40.

7. Mulsow J, Feeley T, Tierney S (2012) Beyond consent-improving understanding in surgical patients. Am J Surg 203: 112-120.

8. Kessels R (2003) Patients' memory for medical information. J R Soc Med 96 219-222.

9. Jones B, Ying W (2005) Using computerized clinical practice guidelines to generate tailored patient education materials. J Am Med Inform Assoc 1: 1-10.

10. Rimer B, Kreuter M (2006) Advancing tailored health communication: A persuasion and message effects perspective. J Communication 56: 184-201.

11. Astley C, Chew D, Aylward P, Molloy D, De Pasquale C (2008) A Randomised study of three different informational aids prior to coronary angiography, measuring patient recall, satisfaction and anxiety. Heart Lung Circ 17: 25-32. 
Citation: Mojadam AN, Nadeem N, Beydoun H, Abidi SR, Rizvi A, et al. (2018) Personalized Preoperative Education System to Assist Patients Undergoing TAVI Surgery: A Digital Health Solution. J Health Med Informat 9: 313. doi: 10.4172/2157-7420.1000313

12. Nguyen T, Attkisson C, Stegner B (2005) Assessment of patient satisfaction: Development and refinement of a Service Evaluation Questionnaire. Eval Program Plann 6: 299-313.

13. Wu K, Chen S, Ko W, Kuo S, Chen P, et al. (2013) The effectiveness of an accessibility-enhanced multimedia informational educational programme in reducing anxiety and increasing satisfaction of patients undergoing cardiac catheterisation. J Clin Nurs 23: 2063-2073.

14. Aitini, Enrico (2014) Communication models for doctor-patient relationships. J Cancer Educ 29: 211-212.

15. Guo P (2014) Preoperative education interventions to reduce anxiety and improve recovery among cardiac surgery patients: a review of randomised controlled trials. J Clin Nurs 24: 34-46.

16. Pereira L, Figueiredo-Braga M, Carvalho I (2016) Preoperative anxiety in ambulatory surgery: The impact of an empathic patient-centered approach on psychological and clinical outcomes. Patient Educ Couns 99: 733-738.

17. Levit, Laura A (2013) Delivering high-quality cancer care: Charting a new course for a system in crisis.

18. Chen J, Mullins C, Novak P, Thomas S (2015) Personalized strategies to activate and empower patients in health care and reduce health disparities. Health Educ Behav 43: 25-34.
19. Riaño D (2008) Knowledge management for health care procedures.

20. Yan A, Qi W (2016) Introduction to Intelligent Surveillance.

21. Syed A, Zeng $Y$ (2006) Intelligent information personalization leveraging constraint satisfaction and association rule methods. Lect Not Compu Sci 1: 134-145.

22. Han L (2016) Rule based systems for big data: A machine learning approach.

23. Panchal D, Shah S (2011) Artificial intelligence based expert system for Hepatitis B diagnosis. Int J Model Optimiz 1: 362-366.

24. Gath SJ, Kulkarni RV (2012) A review: Expert system for diagnosis of myocardial infarction. Int J Compu Sci Informat Technolog 3: 1-7.

25. Dimytri S, Alexandre A, Magaly A, Eduardo S (2012) Transcatheter aortic valve replacement in elderly patients. J Geriatr Cardiol 9: 78-82.

26. Sardar P, Kundu A, Chatterjee S, Feldman D, Owan T, et al. (2017) Transcatheter versus surgical aortic valve replacement in intermediate-risk patients: Evidence from a meta-analysis. Catheter Cardiovasc Interv 90: 504-515.

27. Elmaraezy A, Ismail A, Abushouk A, Eltoomy M, Saad S, et al. (2017) Efficacy and safety of transcatheter aortic valve replacement in aortic stenosis patients at low to moderate surgical risk: a comprehensive meta-analysis. BMC Cardiovasc Disord 17: 234 